\title{
Bounds on the Growth of the Support of a Vortex Patch
}

\author{
Carlo Marchioro \\ Dipartimento di Matematica, Università “La Sapienza," Piazzale A. Moro 2, I-00185 Roma, Italy
}

Received: 2 April 1993

\begin{abstract}
We study the time evolution of the support of a vortex patch evolving in $\mathbb{R}^{2}$ according to the Euler Equation for an incompressible fluid and we bound its growth. Furthermore we discuss the same problem in the framework of a simplified model. Finally we consider a similar problem for the Navier-Stokes flow.
\end{abstract}

\section{Introduction}

In this paper we study the behavior of a non-viscous incompressible fluid in $\mathbb{R}^{2}$. In particular we consider the so-called vortex patch, that is a system in which the vorticity $\omega(x, 0)$ is proportional to the characteristic function $\chi$ of a region $\Lambda_{0}$ :

$$
\omega(x, 0)=a \chi\left(\Lambda_{0}\right) \quad a \in \mathbb{R} .
$$

We suppose that initially $\Lambda_{0}$ has a bounded diameter $2 R_{0}$. Then we evolve the vorticity by means of the Euler Equations: $\omega(x, 0) \rightarrow \omega(x, t)$. As it is well known $\omega(x, t)$ has the form:

$$
\omega(x, t)=a \chi\left(\Lambda_{t}\right)
$$

Denote by $2 R_{t}$ the diameter of $\Lambda_{t}$. We want to control its growth in time. This problem is interesting both for theoretical reasons, to understand deeply the dynamical behavior of the Euler equation and for applied ones (see for instance pollution problems).

In the present paper we want to find $\alpha, b$ for which we have:

$$
R_{t} \leq\left(R_{0}^{1 / \alpha}+b t\right)^{\alpha} \text { for } t \geq 0 .
$$

It is trivial to observe that the boundedness of the velocity of the flow particles assures that Eq. (1.3) holds with $\alpha=1$; however this bound is very bad and not so interesting. We want to improve this estimate. We observe that the main part of the

^ Research supported by MURST and by CNR-GNFM 
vorticity remains in a bounded region (as consequence of stability results for circular vortex patch, see [WaP85, MaP85, Dri88]), but thin filaments have a complicate motion and may be pushed away. On a particle of the extreme part of the filament mainly act two fields: the first is due to the vorticity near the center, the second is due to the vorticity contained in a region close to the particle. The first one is easily controlled and gives a velocity field less than a constant times $R_{t}^{-2}$. The second is more complicated as we shall see later. In any case, also if we neglect the last term, we cannot obtain a bound better than

$$
\frac{d}{d t} R_{t} \leq \frac{\text { constant }}{R_{t}^{2}}
$$

which implies Eq. (1.3) with $\alpha=\frac{1}{3}$. This seems the optimal estimate that we can find using the general argument only. In the present paper we prove exactly this bound.

This result is valid for any initial configuration. For particular initial data the bound on $R_{t}$ might be better. Actually different parts of the vortex patch could turn around with different speeds so that a sort of homogenization happens asymptotically in time. As a consequence, the radial velocity decreases in time faster as the circular symmetry is reached. Moreover, when a particle of a filament is far from the center of vorticity, it moves slowly, so that the freedom degrees of particles near the center are "fast variables" for it. Their action can be averaged on a large interval of time. This is a further effect of homogenization (temporal homogenization). An example of this effect arises in the so-called Kirchhoff ellipse (see the appendix). In conclusion, if the homogenization happens, it is natural to suppose that $\alpha \ll \frac{1}{3}$. We believe that the main part of the initial condition gives rise to some homogenization, but it is very difficult to prove it and to exclude that some "resonant" situation could happen for which bound (1.3), with $\alpha=\frac{1}{3}$, is the best possible. In the next section we obtain bounds valid for any initial data and we do not take care of this interesting but difficult phenomenon. In Sect. 3 we discuss a simplified model more easy to investigate that exhibits this homogenization effect. For numerical pictures see [Dri89] and references quoted therein.

Finally in Sect. 4 we study the problem for a Navier-Stokes flow. In this case the viscosity produces an immediate diffusion and the vorticity has an unbounded support. However we can study the growth of a region out of which the vorticity mass is exponentially negligible. We find a bound analogous to that obtained in the pure diffusive case.

\section{Euler Flow}

Consider an incompressible non-viscous fluid of unitary density moving in $\mathbb{R}^{2}$. The Euler Equation in term of the vorticity reads:

$$
\begin{gathered}
\partial_{t} \omega(x, t)+(u \cdot \nabla) \omega(x, t)=0 \\
\nabla \cdot u(x, t)=0, \\
\omega \equiv \operatorname{curl} u=\partial_{1} u_{2}-\partial_{2} u_{1}, \quad \omega(x, t)=\omega_{0}, \quad x \equiv\left(x_{1}, x_{2}\right) \in \mathbb{R}^{2} .
\end{gathered}
$$

Here $u=\left(u_{1}, u_{2}\right)$ denotes the velocity field. 
If $u$ decays at infinity, as we suppose in this paper, we can reconstruct the velocity field by means of $\omega$ as

$$
\begin{gathered}
u(x, t)=\int K(x-y) \omega(x, t) d y, \\
K=\nabla^{\perp} G(x),
\end{gathered}
$$

where

$$
\nabla^{\perp} \equiv\left(\partial_{2},-\partial_{1}\right)
$$

and

$$
G(x)=-\frac{1}{2 \pi} \ln |x|
$$

As well known, Eq. (2.1) means that the vorticity is constant along the particle paths, which are the characteristic of the Euler Equation. Therefore

$$
\omega(x, t)=\omega\left(x_{0}(x,-t), 0\right),
$$

where the trajectory $x\left(x_{0}, t\right)$ of the fluid particle initially in $x_{0}$ satisfies:

$$
\begin{gathered}
\frac{d}{d t} x\left(x_{0}, t\right)=u\left(x\left(x_{0}, t\right), t\right), \quad x\left(x_{0}, 0\right)=x_{0}, \\
u(x, t)=\int K(x-y) \omega(x, t) d y .
\end{gathered}
$$

We want to study the Euler Equation when the initial data have the form

$$
\omega_{0}=a \chi\left(\Lambda_{0}\right), \quad a \in \mathbb{R}
$$

and $\chi\left(\Lambda_{0}\right)$ denotes the characteristic function of $\Lambda_{0} \subset \mathbb{R}^{2}$. Then we need a weak formulation of Eqs. (2.1), (2.2), (2.3) which is given by Eqs. (2.8), (2.9), (2.10) or by the equivalent form

$$
\frac{d}{d t} \omega_{t}[f]=\omega_{t}[u \cdot \nabla f]
$$

where $f$ is a smooth function and

$$
\omega_{t}[f] \equiv \int f(x) \omega(x, t) d x
$$

In conclusion

$$
\omega(x, t)=a \chi\left(\Lambda_{t}\right)
$$

where $\Lambda_{t}$ is the time evolution of $\Lambda_{0}$ by Eqs. (2.9), (2.10), i.e.

$$
x\left(x_{0}, t\right) \in \Lambda_{t} \quad \text { iff } \quad x_{0} \in \Lambda_{0} .
$$

As well known the Lebesgue measure of $\Lambda_{t}$ is equal to the measure of $\Lambda_{0}$. (For the standard results on the Euler Equation see, for instance, [MaP94]).

We prove the following result:

Theorem 2.1. Suppose that

$$
\Lambda_{0} \subset \Sigma\left(R_{0}\right)
$$


where $\Sigma(R)$ in the circle with center in the origin and radius $R$. Then for any $\alpha \geq \frac{1}{3}$ there is a constant $b>0$ such that

$$
\Lambda_{t} \subset \Sigma\left(R_{t}\right)
$$

with

$$
R_{t} \leq\left(R_{0}^{1 / \alpha}+b t\right)^{\alpha} \text { for } t \geq 0
$$

(Of course the sharpest bound is obtained when $\alpha=\frac{1}{3}$.)

The technique of the proof is inspired to the papers [Mar88, MaP92], developed however in a different context.

The strategy of the proof is the following: we find an upper bound for the radial component of the velocity field $u_{r}$ computed in a point $x$ such that $|x|=R_{t}$. In particular we prove that

$$
\left|u_{r}\right| \leq R_{t}^{-\beta}
$$

(from now on $C$ denotes a positive constant). Then

$$
\frac{d}{d t} R_{t} \leq C R_{t}^{-\beta}
$$

This differential inequality implies Eq. (2.18) with

$$
\alpha=\frac{1}{1+\beta} \text {. }
$$

We remark that the bound (2.18) becomes more sharp as $\alpha$ decreases (i.e. $\beta$ increases). So $\alpha$ must be as small as possible. We shall see that for $\alpha=\frac{1}{2}$ (i.e. $\beta=1$ ) the proof is quite simple, while the general case is more inolved.

Proof for $\alpha=\frac{1}{2}$.

From now on for simplicity we suppose $a=1$ and meas $\Lambda_{0}=1$.

We write

$$
u_{r}=\frac{x}{|x|} \cdot u(x, t)=\frac{x}{|x|} \cdot \int K(x-y) \omega(x, t) d y=\frac{x}{|x|} \cdot \int_{\Lambda_{t}} K(x-y) d y .
$$

Hence

$$
\left|u_{r}\right| \leq\left|\int_{\Lambda_{t}} K(x-y) d y\right|
$$

We divide the integration domain $\Lambda_{t}$ into two sets:

$$
A_{t}=\Sigma\left(\frac{R_{t}}{2}\right) \cap \Lambda_{t}
$$

and

$$
A_{2}=\Sigma^{c}\left(\frac{R_{t}}{2}\right) \cap \Lambda_{t}
$$

(where $\Sigma^{c}$ is the complement of $\Sigma$ ). Then

$$
\int_{\Lambda_{t}} K(x-y) d y=\int_{A_{1}}+\int_{A_{2}}
$$


The first integral is easily evaluated by using the obvious inequality

$$
|K(x)| \leq C|x|^{-1}
$$

Hence

$$
\left|\int_{A_{1}} K(x-y) d y\right| \leq \frac{C}{R_{t}} .
$$

To evaluate the second integral we observe that a bound on it is obtained considering the integral performed on a circular domain centered on $x$ and with area equal to meas $A_{2}$ :

$$
\left|\int_{A_{2}} K(x-y) d y\right|<\left|\int_{\eta} K(-y) d y\right|=\frac{1}{2 \pi} \int_{0}^{2 \pi} d \theta \int_{0}^{\eta} \frac{1}{|y|} d y=\eta,
$$

where

$$
\pi \eta^{2}=\text { meas } A_{2} \Rightarrow \eta=\sqrt{\frac{\text { meas } A_{2}}{\pi}} .
$$

We denote by $m_{t}(r)$ the vorticity out of $\Sigma(r)$ at time $t$. Then we use the fact that the quantity

$$
I=\int \omega(x, t) x^{2} d x \quad \text { (moment of inertea) }
$$

is conserved during the motion (as can be verified by direct computation).

We have trivially

$$
I \geq r^{2} m_{t}(r)
$$

which implies

$$
m_{t}(r) \leq C r^{-2}
$$

Observing that $m_{t}\left(\frac{R}{2}\right)=$ meas $A_{2}$, inserting Eq. (2.33) in Eq. (2.29), from Eq. (2.23) we have

$$
\left|u_{r}\right| \leq C \frac{1}{R_{t}}
$$

that is Eq. (2.19) with $\beta=1$ (i.e. $\alpha=\frac{1}{2}$ ).

Proof of Theorem 2.1. Define

$$
r_{t}=\left(R_{0}^{3}+b_{1} t\right)^{1 / 3}
$$

where $b_{1}$ shall be fixed later.

Let $t^{*}>0$ a time such that for any $t, 0 \leq t \leq t^{*}$, the inequality $R_{t} \leq r_{t}$ holds. $\left(t^{*}\right.$ is surely larger than zero for $b_{1}$ large because of the boundness of the velocity field). Then we prove in $|x|=r_{t}$ a bound like (2.19) with $\beta=2$ with $\beta=2$ and so a bound like (2.18) with $\alpha=\frac{1}{3}$ with the same $b_{1}$. In the proof $b_{1}$ is obtained independent of $t^{*}$. Hence $t^{*} \rightarrow \infty$ and the theorem is proved for $b=b_{1}$. 
To evaluate the radial velocity field in $x,|x|=r_{t}$, we divide the circle $\Sigma\left(r_{t}\right)$ into many different annulii:

$$
\Sigma\left(r_{t}\right)=\sum_{k=1}^{k^{*}}\left[\Sigma\left(a_{k}\right)-\Sigma\left(a_{k-1}\right)\right] \cup\left[\Sigma\left(r_{t}\right)-\Sigma\left(a_{k^{*}}\right)\right]
$$

where

$$
\begin{gathered}
a_{0}=0, \quad a_{1}=R_{0}, \quad a_{k}=2 a_{k-1}, \\
k^{*} \text { is such that } a_{k^{*}+1} \leq r_{t} \text { and } a_{k^{*}+2}>r_{t} .
\end{gathered}
$$

The radial velocity in $x$ in given by Eq. (2.22). It can be expressed by the sum of the contributions obtained when the vorticity is contained in each annulus:

$$
\begin{aligned}
& \frac{x}{|x|} \cdot \int_{\left[\Sigma\left(a_{k}\right)-\Sigma\left(a_{k-1}\right)\right] \cap \Lambda_{t}} K(x-y) d y \\
& \quad=\frac{x}{|x|} \cdot \int_{\left[\Sigma\left(a_{k}\right)-\Sigma\left(a_{k-1}\right)\right] \cap \Lambda_{t}} K(x) d y \\
& \quad+\frac{x}{|x|} \cdot \int_{\left[\Sigma\left(a_{k}\right)-\Sigma\left(a_{k-1}\right)\right] \cap \Lambda_{t}}[K(x-y)-K(x)] d y .
\end{aligned}
$$

The first term in the right-hand side of Eq. (2.38) vanishes because of $x \cdot K(x)=0$. Moreover, by the explicit form of $K(x)$, we have

$$
|K(x-y)-K(x)|<C \frac{\gamma}{r_{t}\left(r_{t}-\gamma\right)} \quad \text { if } \quad|y|<\gamma .
$$

Hence

$$
\begin{aligned}
& \left|\frac{x}{|x|} \cdot \int_{\left[\Sigma\left(a_{k}\right)-\Sigma\left(a_{k-1}\right)\right] \cap \Lambda_{t}}[K(x-y)-K(x)] d y\right| \\
& \quad \leq C \frac{a_{k}}{r_{t}\left(r_{t}-a_{k}\right)} \mid \int_{\left[\Sigma\left(a_{k}\right)-\Sigma\left(a_{k-1}\right)\right] \cap \Lambda_{t}} d y
\end{aligned}
$$

(by estimate $(2.33)$ )

$$
\leq \frac{C}{a_{k} r_{t}\left(r_{t}-a_{k}\right)}=\frac{C}{2^{k-1} r_{t}\left(r_{t}-2^{k-1} R_{0}\right)}
$$

We put Eq. (2.40) into Eq. (2.38) and we have

$$
\sum_{k=1}^{k^{*}}\left|\frac{x}{|x|} \cdot \int_{\left[\Sigma\left(a_{k}\right)-\Sigma\left(a_{k-1}\right)\right] \cap \Lambda_{t}} K(x-y) d y\right| \leq \frac{C}{r_{t}^{2}} \sum_{k=1}^{k^{*}} 2^{-k} \leq \frac{C}{r_{t}^{2}} .
$$

It remains to evaluate the integral (2.22) in the domain $\left[\Sigma\left(r_{t}\right)-\Sigma\left(a_{k^{*}}\right)\right] \cap \Lambda_{t}$. It is easy to do in the region in which the integrand is bounded. In fact its contribution is smaller than $\mathrm{Cr}_{t}^{-2}$, as it follows from estimate (2.33) which reads

$$
m_{t}\left(a_{k^{*}}^{-2}\right)<C a_{k^{*}}^{-2}
$$


and the obvious observation that $a_{k^{*}}>\frac{r_{t}}{4}$. When the integrand becomes unbounded we need a more accurate estimate on $m_{t}\left(a_{k^{*}}\right)$. To evaluate $m_{t}\left(a_{k^{*}}\right)$ at time $t$, we prove that it depends on $m_{t}\left(a_{k^{*}}-R_{0}\right)$ at a time before and so on, starting an iterative procedure. More precisely we introduce a function $W_{R} \in C^{\infty}\left(\mathbb{R}^{2}\right), r \rightarrow W_{R}(r)$ depending only on $|r|$, not increasing in $|r|$,

$$
W_{R}(r)=\begin{array}{lll}
1 & \text { if } \quad|r| \leq R \\
0 & \text { if } \quad|r|>R+R_{0}
\end{array},
$$

and such that for some $C_{1}>0$,

$$
\begin{gathered}
\left|\nabla W_{R}(r)\right| \leq C_{1}, \\
\left|\nabla W_{R}(r)-\nabla W_{R}\left(r^{\prime}\right)\right| \leq C_{1}\left|r-r^{\prime}\right| .
\end{gathered}
$$

Then we define a smooth version of $m_{t}(R)$ :

$$
\mu_{t}(R)=1-\int W_{R}(x) \omega(x, t) d x
$$

Hence, using the Euler equation (2.12), we have:

$$
\begin{aligned}
\frac{d}{d t} \mu_{t}(R) & =-\int_{\Lambda_{t}} d x u(x, t) \cdot \nabla W_{R}(x) \\
& \left.=-\int_{\Lambda_{1}} d x \int_{\Lambda_{t}} d y \nabla W_{R}(x) \cdot K(x-y) \text { (by the antisymmetry of } K\right) \\
& =-\frac{1}{2} \int_{\Lambda_{t}} d x \int_{\Lambda_{t}} d y\left[\nabla W_{R}(x)-\nabla W_{R}(y)\right] \cdot K(x-y) .
\end{aligned}
$$

We want to estimate this term for $R=n R_{0}, n>1$. We divide the integration domain into the following sets:

$$
\begin{aligned}
T_{h}= & \left\{(x, y) \in \Lambda_{t} \times \Lambda_{t} \mid x \notin \Sigma\left(n R_{0}\right), y \in\left[\Sigma\left(a_{h}\right)-\Sigma\left(a_{h-1}\right)\right]\right\} \\
& \text { if } h<k, \\
T_{h}= & \left\{(x, y) \in \Lambda_{t} \times \Lambda_{t} \mid x \notin \Sigma\left(n R_{0}\right), y \notin \Sigma\left(a_{k-1}\right)\right\} \\
& \text { if } h=k \\
S_{h}= & \left\{(x, y) \in \Lambda_{t} \times \Lambda_{t} \mid y \notin \Sigma\left(n R_{0}\right), x \in\left[\Sigma\left(a_{h}\right)-\Sigma\left(a_{h-1}\right)\right]\right\} \\
& \text { if } h<k, \\
S_{h}= & \left\{(x, y) \in \Lambda_{t} \times \Lambda_{t} \mid y \notin \Sigma\left(n R_{0}\right), x \notin \Sigma\left(a_{k-1}\right)\right\} \\
& \text { if } h=k,
\end{aligned}
$$

where $a_{h}$ are defined in Eq. (2.37).

We choose $k$ such that $a_{k+1} \leq n R_{0}$ and $a_{k+2}>n R_{0}$.

Notice that the integrand in Eq. (2.47) vanishes in the complement of $\bigcup_{h=1}^{k}\left(T_{h} \cup S_{h}\right)$.

Thanks to the identity $\nabla W_{n R_{0}}(x) \cdot K(x)=0$ and the fact that $\nabla W_{n R_{0}}(y)=0$ if $y \in\left[\Sigma\left(a_{h}\right)-\Sigma\left(a_{h-1}\right)\right], h<k$, the contribution on the integral (2.47) due to $T_{h}$, 
$h<k$ is bounded by

$$
\begin{aligned}
& \frac{1}{2}\left|\int_{\Lambda_{t}} d x \int_{\Lambda_{t} \cap\left[\Sigma\left(a_{h}\right)-\Sigma\left(a_{h-1}\right)\right]} d y \nabla W_{n R_{0}}(x) \cdot[K(x-y)-K(y)]\right| \\
& \quad \leq \text { (by }(2.44), \text { the fact that } \nabla W_{n R_{0}}(x)=0 \text { if }|x|<n R_{0} \text { and inequality (2.39)) } \\
& \quad \leq C \frac{2^{h}}{\left(n R_{0}\right)^{2}}\left[m_{t}\left(\Sigma\left(a_{h}\right)\right)-m_{t}\left(\Sigma\left(a_{h-1}\right)\right)\right] m_{t}\left(n R_{0}\right) \leq \text { by (2.33)) } \\
& \quad \leq C \frac{m_{t}\left(n R_{0}\right)}{\left(n R_{0}\right)^{2} 2^{h}}
\end{aligned}
$$

To estimate the contribution due to $T_{k}$, we use the obvious inequality $|K(x)| \leq$ $C|x|^{-1}$, Eq. (2.45) and the bound

$$
\left|\left\{\nabla W_{R}(x)-\nabla W_{R}(y)\right\} \cdot K(x-y)\right| \leq C,
$$

and we obtain that it is smaller than

$$
C \frac{m_{t}\left(n R_{0}\right)}{\left(n R_{0}\right)^{2}}
$$

In conclusion, let $A_{3}=\bigcup_{h=1}^{k} T_{h}$, then

$$
\begin{aligned}
& \left|\int_{A_{3}} d x d y\left[\nabla W_{n R_{0}}(x)-\nabla W_{n R_{0}}(y)\right] \cdot K(x-y)\right| \\
& \quad \leq C \frac{m_{t}\left(n R_{0}\right)}{\left(n R_{0}\right)^{2}} \sum_{h=1}^{k} 2^{-h}<C \frac{m_{t}\left(n R_{0}\right)}{\left(n R_{0}\right)^{2}} .
\end{aligned}
$$

The terms due to $\bigcup_{h=1}^{k} S_{h}$ can be handed exactly in the same way. Hence

$$
\left|\frac{d}{d t} \mu_{t}\left(n R_{0}\right)\right| \leq C \frac{m_{t}\left(n R_{0}\right)}{\left(n R_{0}\right)^{2}} .
$$

We observe now that trivially

$$
m_{t}\left(n R_{0}\right) \leq \mu_{t}\left((n-1) R_{0}\right)
$$

Hence the identity

$$
\mu_{t}\left(n R_{0}\right)=\mu_{0}\left(n R_{0}\right)+\int_{0}^{t} d \tau \frac{d}{d \tau} \mu_{\tau}\left(n R_{0}\right)
$$

gives, by Eqs. (2.56), (2.57),

$$
\mu_{t}\left(n R_{0}\right) \leq C \int_{0}^{t} d \tau \frac{\mu_{t}\left((n-1) R_{0}\right)}{\left(n R_{0}\right)^{2}}
$$

because $\mu_{0}\left(n R_{0}\right)=0$ for $n \geq 1$. 
We are now able to apply an iterative procedure.

We start from $n=2^{k^{*}-1}-1$ and we arrive at 1 . We apply many times Eq. (2.59) and then Eq. (2.57). We have

$$
m_{t}\left(a_{k^{*}}\right) \leq \frac{C^{n} t^{n}}{(n !)^{3}}
$$

Since

$$
t<r_{t}^{3} b_{1}^{-3}
$$

and

$$
r_{t}<C n
$$

using the Stirling formula

$$
\ln n !>n(\ln n-1)
$$

we have

$$
m_{t}\left(a_{k^{*}}\right)<\frac{C^{n} n^{3 n}}{b_{1}^{n}(n !)^{3}}<C^{n} b_{1}^{-n} .
$$

We choose $b_{1}$ large enough (independently of $n$ ) to obtain

$$
m_{t}\left(a_{k^{*}}\right)<C^{n} b_{1}^{-n}<C_{2} n^{-4} .
$$

We have so obtained an accurate bound on $m_{t}\left(a_{k^{*}}\right)$. To evaluate the radial field in $x,|x|=r_{t}$, due to the vorticity out of $\Sigma\left(a_{k^{*}}\right)$ we use the same technique of Eq. (2.29) and we prove that it is smaller than

$$
C_{3}\left(a_{k^{*}}\right)^{-2}<C_{4} r_{t}^{-2}
$$

In conclusion adding estimate (2.41) to estimate (2.66), we obtain that the radial velocity field in $x,|x|=r_{t}$ is smaller than

$$
\frac{C+C_{4}}{r_{t}^{2}}
$$

with $C$ independent of $b_{1}$ and $C_{4}$ decreasing as $b_{1}$ increases. Equation (2.67) gives a bound like (2.18) with $\alpha=\frac{1}{3}$ and $b=3\left(C+C_{4}\right)$. So we can choose $b_{1}$ large such that $b_{1}>3\left(C+C_{4}\right)$, and then $R_{t} \leq r_{t}$ for any time.

In Theorem $2.1 b$ depends, of course, on the initial conditions. It is natural to prove that $b \rightarrow 0$ as the initial vortex patch becomes circular. More precisely:

Theorem 2.3. Let $b$ defined by Eq. (2.18). Then

$$
b \rightarrow 0 \text { as } I \rightarrow I_{0},
$$

where $I$ is the moment of inertia defined by Eq. (2.18) and $I_{0}=(2 \pi)^{-1}$ is the moment of inertia of a circular vortex patch of unitary mass.

Proof. The proof is based on an improvement of the bound (2.33).

With fixed $m_{t}(r)$, we observe that the configuration which gives a minimal $I$ is the following: $\Lambda_{0}$ is the sum of two regions, the first consisting of an annulus of interior radius $r$ and area $m_{t}(r)$, the second one consisting in a circle of area $1-m_{t}(r)$. Then

$$
I \geq 2 \pi \int_{r}^{r_{1}} r^{3} d r+2 \pi \int_{0}^{r_{2}} r^{3} d r,
$$


where $r_{1}$ and $r_{2}$ are defined by the relations

$$
\begin{aligned}
2 \pi \int_{r}^{r_{1}} r d r & =m_{t}(r), \\
2 \pi \int_{0}^{r_{2}} r d r & =1-m_{t}(r) .
\end{aligned}
$$

Hence

$$
\begin{aligned}
I & \geq \frac{\pi}{2}\left\{\left[\frac{m_{t}(r)}{\pi}+r^{2}\right]^{2}-r^{4}+\left[\frac{1-m_{t}(r)}{\pi}\right]^{2}\right\} \\
& =\frac{m_{t}(r)^{2}}{\pi}+m_{t}(r)\left(r^{2}-\frac{1}{\pi}\right)+\frac{1}{2 \pi}
\end{aligned}
$$

and so

$$
m_{t}(r) \leq \frac{I-(2 \pi)^{-1}}{r^{2}-\pi^{-1}}
$$

Obviously $r \geq R_{0}>(\pi)^{-1 / 2}$ and so that bound (2.33) holds with a constant vanishing as $I \rightarrow I_{0}$. Since this bound appears as a multiplicative factor in all the later steps, $b$ also vanishes as $I \rightarrow I_{0}$.

Remark. Until now we have considered $\omega_{0}$ of the form of a characteristic function of a set but all the previous considerations apply without any change if $\omega_{0}$ is a generic bounded function of definite sign with a compact support.

\section{A Simplified Model}

In the previous section we have neglected completely the effects of the homogenization. The nonlinear nature of the model makes it very difficult to take them into account. To simplify the problem we can introduce some new, more tractable, models, that are schematic approximations of the vortex patch dynamics and which exhibit the homogenization effects. We discuss briefly one of them:

$$
\begin{aligned}
& \omega_{0}=\chi\left(\Lambda_{0}\right), \\
& \Lambda_{0}=\Sigma\left(\frac{1}{\sqrt{\pi}}\right) \cup \Omega_{0} \cup \Xi_{0},
\end{aligned}
$$

where $\Sigma$ is the circle of radius $\frac{1}{\sqrt{\pi}}$ and

$$
\Omega_{0}=\left\{x \in \mathbb{R}^{2}\left|\frac{1}{\sqrt{\pi}} \leq\right| x \mid \leq f(\theta)\right\} \quad(\varrho, \theta)=\text { polar coordinates },
$$
$f(\theta)$ being a smooth periodic function such that $f(\theta) \geq \frac{1}{\sqrt{\pi}}$. Finally $\Xi_{0}$ is a compact
set.

We assume that the initial vorticity $\omega_{0}$ evolves in $\omega_{t}$,

$$
\omega_{t}=\chi\left(\Lambda_{t}\right)
$$


and $\Lambda_{t}$ is obtained from $\Lambda_{0}$ by the following rules: a point of $\Sigma\left(\frac{1}{\sqrt{\pi}}\right) \cup \Omega_{0}$ moves according the velocity field produced by $\Sigma\left(\frac{1}{\sqrt{\pi}}\right)$ alone, while $\Xi_{0}$ evolves in the velocity field produced by $\Sigma\left(\frac{1}{\sqrt{\pi}}\right) \cup \Omega_{0}$ but not by itself.

Of course this model is a first order approximation for $\Omega_{0}$ and $\Xi_{0}$ small of the stationary solution $\Lambda_{0}=\Sigma\left(\frac{1}{\sqrt{\pi}}\right)$.

We can easily prove that this model exhibits the property of spatial homogenization, as we show briefly. In particular we find a bound on the growth of $R_{t}$ :

Theorem 3.1. Suppose

$$
\Lambda_{0} \subset \Sigma\left(R_{0}\right)
$$

then $\Lambda_{t}$

$$
\Lambda_{t} \subset \Sigma\left(R_{t}\right)
$$

where

$$
R_{t} \leq\left(R_{0}^{3}+b \ln (1+t)\right)^{1 / 3} \quad t \geq 0 .
$$

Proof. We only sketch the proof. We evaluate the radial velocity field in the point $x,|x|=R_{t}$. We prove that it is smaller than $C t^{-1}$. Then Eq. (3.8) follows from the integration of the differential inequality (2.20).

The main observation for the proof is the following: points of $\Omega_{0}$ with different $\varrho$ move with different angular velocity. In fact the angular velocity $\gamma$ of a point of radial coordinate $\varrho$ is

$$
\gamma=\frac{1}{2 \varrho^{2}}
$$

Hence during the motion points with different $\varrho$ have different phases and this fact makes it rise to a spatial homogenization.

More precisely, we put ourselves in a reference frame turning around the origin with an angular velocity $\frac{1}{2}$. We denote by

$$
\varrho_{\max }=\max _{0 \leq \theta \leq 2 \pi} f(\theta)
$$

we divide the interval $\left(\varrho_{\max }, 0\right)$ in $N$ disjoint intervals $I_{n}=\left(b_{n}, b_{n-1}\right)$ such that each point in $I_{n}$ has made $n-1$ turns and not $n$ turns. We study the size of $I_{n}$. We compute the angular velocity $\gamma$ corresponding to extremal points $b_{n}$ and $b_{n-1}$. We have

$$
\begin{aligned}
\gamma\left(b_{n}\right) & =\frac{2 \pi}{t} n, \\
\gamma\left(b_{n-1}\right) & =\frac{2 \pi}{t}(n-1) .
\end{aligned}
$$

Hence

$$
\left|\gamma\left(b_{n}\right)-\gamma\left(b_{n-1}\right)\right|=\frac{2 \pi}{t} .
$$

In $I_{n}$ the angular velocity changes linearly up to a correction of order $t^{-2}$, so that the size of $I_{n}$ is of the order of $t^{-1}$.

Moreover it is easy to see that the particles with $\varrho \in I_{n}$ are uniformly distributed in the corresponding annulus up to a correction of the order $t^{-1}$. Of course a circular 
distribution cannot produce a radial velocity field. Then the radial velocity field produced by the particles with $\varrho \in I_{n}$ on a particle in $x,|x|=R_{t}$ can be bounded by:

$$
\leq \frac{C}{R_{t}^{2}} \frac{\mu\left(I_{n}\right)}{1+t}
$$

where $\mu\left(I_{n}\right)$ is the measure of the set of $\Omega_{0}$ corresponding to $\varrho \in I_{n}$, which is constant during the motion because the velocity field is divergence-free.

By summing on $n$, we have

$$
\frac{d}{d t} R_{t} \leq \frac{C}{R_{t}^{2}} \frac{1}{1+t}
$$

Hence, by integration of this differential inequality, we obtain Eq. (3.8).

We do not know if bound (3.8) is the best possible in this model. In fact we have not fully used the temporal homogenization, when we have neglected an overall angular motion. To take into account this temporal homogenization effect we need to consider the sign of the radial velocity variable in time and this is a difficult task. However we notice that for practical purposes bound (3.8) is undistinguishable from a constant bound. Moreover this model, perhaps useful for intermediate times, falls surely asymptotically in time, when the nonlinear effect may become relevant.

\section{Navier-Stokes Flow}

In this section we study the time evolution in $\mathbb{R}^{2}$ of a vortex patch, when the viscosity is present. In this case the Euler equation (2.12) modifies in the NavierStokes equation:

$$
\frac{d}{d t} \omega_{t}[f]=\omega_{t}\left([u \cdot \nabla f]+\nu \omega_{t}[\Delta f] \quad \nu>0 .\right.
$$

Obviously, adding a viscosity term the asymptotic in time behavior of the fluid changes drastically. In fact the fluid diffuses immediately in the whole plane and so an equivalent of Theorem 2.1 is meaningless. However we can study the region $\Sigma\left(r_{t}\right)$ in which the main part of the vorticity is concentrated and bounds its growth in time (that is the equivalent of Theorem 2.3). More precisely we want to find for which $\alpha>0$ we have

$$
\begin{array}{ll}
m_{t}\left(r_{t}\right)<C \exp \left[-\gamma r_{t}^{\delta}\right] & \gamma, \delta>0, \\
\text { if } \quad r_{t}>C t^{\alpha} & t>0 .
\end{array}
$$

(Of course the bound is sharper as $\alpha$ is smaller.) For the Stokes equation (when the transport term $\omega_{t}([u \cdot \nabla f]$ is absent) the problem is easily solved by the explicit solution and we find that the inequality (4.2) holds for any $\alpha>\frac{1}{2}$. In this section we prove that we obtain the same result when we consider the whole Navier-Stokes equation:

Theorem 4.1. Suppose that

$$
\omega_{0}=\chi\left(\Lambda_{0}\right)
$$

with

$$
\Lambda_{0} \subset \Sigma\left(R_{0}\right) \quad R_{0}>0
$$


Then for any $\alpha>\frac{1}{2}, 0<\delta<2-\alpha^{-1}$ we have

$$
m_{t}\left(r_{t}\right)<C \exp \left[-\gamma r_{t}^{\delta}\right] \quad \gamma>0 \text { if } r_{t}>C t^{\alpha},
$$

where $m_{t}(r)$ denotes the vorticity mass at time $t$ out of a circle of radius $r$ and the fluid evolves according to the Navier-Stokes Equation (4.1).

Proof. The proof is based on the following result:

Lemma 4.1. Define

$$
I_{n}(t)=\int_{\mathbb{R}^{2}} d x|x|^{2 n} \omega(x, t) .
$$

Then

$$
\left|\frac{d}{d t} I_{n}(t)\right| \begin{array}{ll}
=0 & \text { if } n=0 \\
\leq C n^{2} I_{n-1}(t) & \text { if } n \geq 1
\end{array}
$$

and hence

$$
I_{n}(t) \leq \sum_{k=1}^{n} C^{k} \frac{(n !)^{2} t^{k}}{[(n-k) !]^{2} k !} .
$$

Proof of the Lemma. The inequality (4.7) can be proved by direct computation. In fact, using Eq. (4.1), we have:

$$
\frac{d}{d t} I_{n}(t)=A+B
$$

where

$$
\begin{aligned}
& A=\int d x \omega(x, t) u \cdot \nabla|x|^{2 n}, \\
& B=\nu \int d x \omega(x, t) \Delta|x|^{2 n},
\end{aligned}
$$

and so the proof of Eq. (4.7) for $n=0$ is trivial. For $n \geq 1$ we study the two terms $A, B$ separately. The second one can be explicitly computed, remembering that in polar coordinates $(r, \theta)$ we have: $\Delta f=r^{-1} \partial_{r}\left(r \partial_{r} f\right)+r^{-2} \partial_{\theta}^{2} f$. We obtain:

$$
B=\nu(2 n)^{2} I_{n-1}(t) \text {. }
$$

Then we study term $A$, using the expression of the velocity field given by Eq. (2.4) and the antisymmetry of $K$,

$$
\begin{aligned}
A= & \int d x \int d y \omega(x, t) \omega(y, t) K(x-y) \cdot \nabla|x|^{2 n} \\
= & \frac{1}{2} \int d x \int d y \omega(x, t) \omega(y, t)\left\{\nabla_{x}|x|^{2 n}-\nabla_{y}|y|^{2 n}\right\} \cdot K(x-y) \\
= & n \int d x \int d y \omega(x, t) \omega(y, t)\left\{|x|^{2 n-2} x-|y|^{2 n-2} y\right\} \cdot K(x-y) \\
= & \frac{n}{2} \int d x \int d y \omega(x, t) \omega(y, t)\left[\left\{|x|^{2 n-2}-|y|^{2 n-2}\right\}(x+y) \cdot K(x-y)\right. \\
& \left.+\left\{|x|^{2 n-2}+|y|^{2 n-2}\right\}(x-y) \cdot K(x-y)\right]
\end{aligned}
$$


The last term of the sum vanishes, as we can prove using the explicit form of $K$ :

$$
K(x, y)=-\frac{1}{2 \pi} \frac{(x-y)^{\perp}}{|x-y|^{2}} ; \quad x^{\perp} \equiv\left(x_{2},-x_{1}\right) .
$$

Hence

$$
|A| \leq\left.\frac{n}{4 \pi} \int d x \int d y \omega(x, t) \omega(y, t)|| x\right|^{2 n-2}-|y|^{2 n-2} \mid \frac{|x+y|}{|x-y|},
$$

where we have used the well-known fact that $\omega(x, t)$ remains non-negative during the motion. Using the identity

$$
|x|^{2 n-2}-|y|^{2 n-2}=(x-y) \cdot(x+y) \sum_{k=0}^{n-2}|x|^{2 n-4-2 k}|y|^{2 k} \quad n \geq 2,
$$

we have

$$
\begin{aligned}
|A| & \leq \frac{n}{2 \pi} \int d x \int d y \omega(x, t) \omega(y, t)\left(|x|^{2}+|y|^{2}\right) \sum_{k=0}^{n-2}|x|^{2 n-4-2 k}|y|^{2 k} \\
& \leq \frac{n}{\pi} \int d x \int d y \omega(x, t) \omega(y, t) \sum_{k=0}^{n-1}|x|^{2 n-2-2 k}|y|^{2 k}
\end{aligned}
$$

We observe that for any $k \in[0, n-1]$,

$$
|x|^{2 n-4-2 k}|y|^{2 k} \leq|x|^{2 n-2}+|y|^{2 n-2} .
$$

(To prove it, it is enough to put $|y|=\gamma|x|$ and Eq. (4.17) becomes $\gamma^{2 k} \leq 1+\gamma^{2 n-2}$ which is verified for any $\gamma$ and $0 \leq k \leq n-1$.)

Using Eq. (4.17), Eq. (4.16) gives:

$$
|A| \leq \frac{n^{2}}{\pi} \int d x \int d y \omega(x, t) \omega(y, t)\left\{|x|^{2 n-2}+|y|^{2 n-2}\right\}=\frac{2 n^{2}}{\pi} I_{0} I_{n-1}(t) .
$$

Since $I_{0}$ is constant during the motion, adding Eq. (4.18) to Eq. (4.12), we have proved Eq. (4.7).

To obtain Eq. (4.8) we integrate Eq. (4.7) $n$ times:

$$
\begin{aligned}
I_{n}(t) & \leq I_{n}(0)+C n^{2} \int_{0}^{t} d t_{1} I_{n-1}\left(t_{1}\right) \\
& \leq I_{n}(0)+C n^{2} I_{n-1}(0) t+C^{2} n^{2}(n-1)^{2} \int_{0}^{t} d t_{1} \int_{0}^{t_{1}} d t_{2} I_{n-2}\left(t_{2}\right) \\
& \leq \sum_{k=0}^{n} I_{n-k}(0) C^{k} \frac{(n !)^{2}}{[(n-k) !]^{2}} \frac{t^{k}}{k !} .
\end{aligned}
$$


The initial conditions assure that

$$
I_{n-k} \leq I_{0} R_{0}^{2(n-k)}
$$

and so the lemma is proved.

We use the previous lemma to bound $m_{t}\left(r_{t}\right)$. In fact by definition (4.6) we have

$$
I_{n}(t) \geq m\left(r_{t}\right) r_{t}^{2 n}
$$

and hence by Eq. (4.8),

$$
m_{t}\left(r_{t}\right) \leq \frac{1}{r_{t}^{2 n}} \sum_{k=0}^{n} C^{k} \frac{(n !)^{2}}{[(n-k) !]^{2}} \frac{t^{k}}{k !} .
$$

We suppose now that $t \geq 1$ (otherwise the proof of the theorem is trivial). Then

$$
m_{t}\left(r_{t}\right) \leq \frac{C^{n}(n+1) M_{n} t^{n}}{r_{t}^{2 n}}
$$

where

$$
M_{n}=\max _{0 \leq k \leq n} \frac{(n !)^{2}}{[(n-k) !]^{2} k !}
$$

We choose $t>C r_{t}^{1 / \alpha}$. Hence

$$
m_{t}\left(r_{t}\right)<C^{n} n M_{n} r_{t}^{(1 / \alpha-2) n}
$$

We bound $M_{n}$. The maximum of

$$
\frac{(n !)^{2}}{[(n-k) !]^{2} k !}=\frac{n^{2}(n-1)^{2} \ldots(n-k+1)^{2}}{k !}
$$

is reached for $k=k^{*}$ when the term added in the numerator is smaller than $k$, that is the greater $k$ such that

$$
(n-k+1)^{2} \geq k
$$

that is

$$
k^{*}=\text { Integer part of }\left(n+\frac{3}{2}-\left[2 n+\frac{5}{4}\right]^{1 / 2}\right)>n-C \sqrt{n}+C .
$$

We put Eq. (4.28) into Eq. (2.24), we neglect the term $[(n-k) !]^{2}$ and we use the Stirling formula

$$
e^{n(\ln n-1)}<n ! \leq n^{n} .
$$

We obtain

$$
M_{n}<\frac{C^{n} n^{2 n}}{(n-C \sqrt{n})^{n-\sqrt{n}}}<C^{n} n^{n+C \sqrt{n}} .
$$

Putting Eq. (4.29) in Eq. (4.25), we have

$$
m_{t}\left(r_{t}\right)<\exp \left\{C n+n \ln n+C \sqrt{n} \ln n+n\left(\alpha^{-1}-2\right) \ln r_{t}\right\} .
$$


The theorem is proved by choosing

$$
n=\text { Integer part of }\left[C r_{t}^{\delta}\right] \text { with } \delta<2-\alpha^{-1} .
$$

In the previous theorem we have supposed $\omega_{0}$ of the form of a characteristic function of a set, but the statement and the proof remain unchanged if $\omega_{0}$ is a generic bounded function of a definite sign with a compact support. On the contrary for non-compact initial data the statement is similar but the proof changes a little.

Theorem 4.2. Suppose

$$
\omega_{0}(x) \leq C \exp \left\{-\gamma|x|^{\delta}\right\}, \quad \gamma, \delta>0 .
$$

Then

$$
m_{t}\left(r_{t}\right)<C \exp \left[-\gamma^{\prime} r_{t}^{\delta^{\prime}}\right] \quad \gamma^{\prime}>0 \quad \text { if } \quad r_{t}>C t^{\alpha} \quad \text { for any } \quad \delta^{\prime}<\delta<2-\frac{1}{\alpha}
$$

Proof. The proof follows the lines of the previous one and we sketch it only. We observe that

$$
\begin{aligned}
I_{k}(0) & =\int_{\mathbb{R}^{2}} d x|x|^{2 k} \omega_{0}(x)<C \int d x|x|^{2 k} \exp \left\{-\gamma|x|^{\delta}\right\} \\
& =C \gamma^{-(2 k+1) / \delta} \Gamma\left(\frac{2 k+1}{\delta}+1\right) \leq C^{k} k^{2 k / \delta},
\end{aligned}
$$

where $\Gamma(z)$ is the gamma function.

From Eq. (4.22) we have

$$
m_{t}\left(r_{t}\right)<C^{n} \sum_{k=0}^{n} \frac{(n-k)^{2(n-k) / \delta}}{r_{t}^{2(n-k)}} \frac{(n !)^{2}}{[(n-k) !] k !} \frac{t^{k}}{r_{t}^{2 k}} .
$$

We choose

$$
t<C r_{t}^{1 / \alpha}
$$

and

$$
n \approx r_{t}^{\delta^{\prime}}
$$

so that

$$
m_{t}\left(r_{t}\right)<C^{n} \sum_{k=0}^{n} r_{t}^{2\left(\delta^{\prime} / \delta-1\right)(n-k)} \frac{(n !)^{2}}{[(n-k) !]^{2} k !} r_{t}^{(1 / a-2) k}
$$

We proceed as in the previous theorem. We look for the maximum of the addendum in the sum which is reached for $k=k^{*}$, where $k^{*}$ is the greater $k$ for which:

$$
(n-k+1)^{2} \geq k r_{t}^{2 \delta^{\prime} / \delta-1 / \alpha} .
$$

We put $k^{*} \approx n^{\beta}$ and we choose $\delta^{\prime}$ so close to $\delta$ (this is the more difficult case) that $n \approx r^{\delta^{\prime}}$ and $\delta^{\prime}<\delta<2-\alpha^{-1}$ imply $\beta<1$. 
Hence

$$
m_{t}\left(r_{t}\right)<\exp \left\{C n+2 n\left(\frac{\delta^{\prime}}{\delta}-1\right) \ln r_{t}\right\}
$$

and, by using Eq. (4.37), the proof is achieved.

\section{Appendix}

For the sake of completeness we prove that a fluid particle moving in the velocity field generated by a Kirchhoff ellipse [Lam32] remains uniformly in time in a bounded region.

It is well known that a vortex patch with an ellipse shape turns around with a constant angular velocity $\gamma$ without changing its form. We put ourselves in the reference frame in which the ellipse is at rest. In this frame a test particle moves under the action of the velocity field produced by the ellipse plus a tangential velocity of intensity $-\gamma \varrho$ :

$$
u=\nabla^{\perp} \varphi-\nabla^{\perp}\left(\frac{1}{2} \gamma \varrho^{2}\right)
$$

where $\varphi$ is the stream function of the velocity field produced by the ellipse.

It is immediate to verify that the following quantity is conserved during the motion:

$$
\varphi-\frac{1}{2} \gamma \varrho^{2}=\text { const }
$$

Since $\varphi$ diverge at infinity as $\ln \varrho$ it cannot compensate the quadratic term, and so the particle remains in bounded regions.

Acknowledgements. It is a pleasure to thank D. Dritschel for suggesting the problem and E. Caglioti, D. Dritschel, R. Piva, G. Riccardi for useful discussions on the homogenization effect. We thank also the Centre de Physique Théorique, CNRS, Luminy and Université de Provence, where part of this research has been done.

Note added in proof. As a corollary, in the present paper we have proved that a fluid particle, initially in $x_{0}$ and moving via the Euler equation, goes away from the initial position at most: ||$x(t)|-| x_{0}|| \leq$ constant $t^{1 / 3}, t>1$. A stronger result is obtained for the model of Section 3: ||$x(t)|-| x_{0}|| \leq$ constant $\left[\ln (1+t)^{1 / 3}\right], t>1$. Recently Emanuele Caglioti and Carlotta Maffei in the paper "Asymptotic Behaviour of Vortex Patches: A Case of Confinement", Dipartimento di Matematica, Università "La Sapienza", Roma (Italy) Nota Interna 93/22 (1993), Boll. Unione Matematica Italiana (in press) have studied the model of Section 3 and they have proved the confinement of the system when $f(\theta)$ has a finite number of nondegenerate critical points. A deeper analysis of the model is in progress by the same authors. 


\section{References}

[Dri88] Dritschel, D.G.: Nonlinear Stability Bounds for Inviscid, Two-Dimensional, Parallel or Circular Flows with Monotonic Vorticity, and Analogous Three-Dimensional QuasiGeostrophis Flows. J. Fluid Mech. 191, 575-581 (1988)

[Dri89] Dritschel, D.G.: Contour Dynamics and Contour Surgery: Numerical Algorithms for Extended, High-Resolution Modelling of Vortex Dynamics in Two-Dimensional, Inviscid, Incompressible Flows. Computer Phys. Reports 10, 77-146 (1989)

[Lam32] Lamb, H.: Hydrodynamics, 6. ed. Cambridge: Cambridge University Press, 1932

[Mar88] Marchioro, C.: Euler Evolution for Singular Initial Data and Vortex Theory: A Global Solution. Commun. Math. Phys. 116, 45-55 (1988)

[Ma85] Marchioro, C., Pulvirenti, M.: Some Considerations on the Nonlinear Stability of Stationary Planar Euler Flows. Commun. Math. Phys. 100, 343-354 (1985)

[Ma92] Marchioro, C., Pulvirenti, M.: Vortices and localization in Euler flows. Commun. Math. Phys. 154, 49-61 (1993)

[MaP94] Marchioro, C., Pulvirenti, M.: Mathematical theory of incompressible nonviscous fluids. Applied Mathematical Sciences 96, Berlin, Heidelberg, New York: Springer 1994

[WaP85] Wan, Y.H., Pulvirenti, M.: Nonlinear Stability of Circular Vortex Patch. Commun. Math. Phys. 99, 435-450 (1985)

Communicated by J.L. Lebowitz 